$$
\hat{\gamma}
$$


Sobre la empatía

y la introspección

emocional en

los diseñadores

Deyanira Bedolla Pereda 


\section{Sobre la empatía y la introspección emocional en los diseñadores}

\section{Deyanira Bedolla Pereda}

dbedolla@correo.cua.uam.mx | Profesora investigadora de tiempo completo, Departamento de Teoría y Procesos del Diseño, División de Ciencias de la Comunicación y Diseño (DCCD), Universidad Autónoma Metropolitana, unidad Cuajimalpa.

Fecha de recepción: 05 abril 2017

Fecha de aceptación: 21 julio 2017

\section{Resumen}

Este trabajo se centra en la consideración de la empatía como habilidad tanto cognitiva como emocional del ser humano con el fin de que el diseñador identifique y entienda a los usuarios a partir de características humanas compartidas. La empatía para conocer al usuario representa un camino distinto al tradicional, mismo que ha sido externo al individuo y basado en observar y cuestionar al usuario. Con base en el cuestionamiento: ¿Qué reconozco en mí que también puedo reconocer en los demás?. Este trabajo propone la introspección como un primer paso para conducir al estudiante a ser empático, se trata de un enfoque complementario al de las técnicas que tradicionalmente han guiado el conocimiento de los usuarios del diseño que permite comenzar a identificar aspectos humanos complejos, como lo es la dimensión emocional, al fomentar el autoconocimiento personal del alumno como un ser humano que es y de este modo comenzar a proyectar para otros seres humanos semejantes a él.

Palabras clave | introspección; empatía; emociones; afectividad; usuarios. 
SOBRE LA EMPATÍA Y LA INTROSPECCIÓN

EMOCIONAL EN LOS DISEÑADORES

\begin{abstract}
This paper focus on empathy as cognitive and emotional human characteristics, with the aim that designers acknowledge particular human qualities in the self as well as in the user. Empathy, in this particular way represents an alternative approach to guide future designers in understanding the user. Instead of observing and interviewing them we encourage designers to reflect on an introductory question: what can I recognize on me, which I can recognize on others? This paper builds on empathy and introspection to develop a technique that explores complex human processes as the emotional dimension. Its main goal is to enhance self-knowledge in design students as a starting point in the process of designing for other humans.
\end{abstract}

Keywords | introspection; empathy; emotions; affectivity; users. 


\section{Introducción}

El concepto de empatía en el que se basa este trabajo, parte de la consideración de que es una habilidad específica del ser humano que involucra lo cognitivo y lo emocional (Stojiljkovic 2012; Rifkin 2010; Wiseman 2007; Morse 1992) y que dada su naturaleza y características permite a una persona, en este caso al futuro diseñador, conocer y abordar problemáticas de su entorno de una manera significativa a nivel personal.

La empatía es una habilidad importante a nivel humano que permite a un individuo entender, ser sensible al otro, a su sentir y a sus problemáticas. En cierta medida es una postura que si bien busca conectar con otras personas, radica en la persona que empatiza, en este caso en los diseñadores.

Es una habilidad humana que por su naturaleza puede representar en el área del diseño una herramienta de gran relevancia y utilidad en el proceso de enseñanza aprendizaje desde distintas perspectivas. Existen estudios previos en México que ya presentan su utilidad; el trabajo de López y Gómez (2014) buscó conocer si estudiantes de diseño habían tenido experiencias empáticas con sus docentes y la importancia que esto representaba para su motivación y aprendizaje; en un segundo trabajo, López-León (2017) enfatiza los alcances que tiene el concepto de empatía en la educación del diseño, específicamente para mejorar el desempeño docente, en su trabajo presenta una serie de actividades para fomentar un proceso que conduzca al docente a ser empático dentro del aula, dichas actividades identificadas por el autor son las siguientes: escuchar, comprender y alinear; discutir y ajustar; y la disposición y percepción de apertura que el docente sea capaz de mostrar hacia el estudiante; con la identificación de estas actividades el López-León buscó plantear las bases para el desarrollo de un modelo que promueva una docencia empática en las aulas de diseño.

Un enfoque distinto al señalado en los trabajos citados se presenta en este artículo; ya que se parte de la consideración de la empatía como una habilidad humana que por su naturaleza puede representar una herramienta para el diseñador y/o futuro diseñador, y como tal ayude a considerar y reflexionar respecto a las personas que interactúan con un diseño, en el presente y en el futuro. 
SOBRE LA EMPATÍA Y LA INTROSPECCIÓN

EMOCIONAL EN LOS DISEÑADORES

Este planteamiento se lleva a cabo con base en el señalamiento de Brown (2010) en el que menciona a la empatía como uno de los requerimientos actuales para el profesional del diseño, subrayando que es necesario que el futuro diseñador cuente con un "gen de la empatía", de servicio a los demás, de manera que sea capaz de imaginar el mundo desde otra perspectiva: la del usuario. Según Brown (2010) en la actualidad es necesario que el diseñador sea capaz de empatizar profundamente con contextos, problemáticas, situaciones, y con sus actores y participantes para el desarrollo del diseño centrado en el usuario. La temática del conocimiento del usuario es una de las materias centrales a considerar en el área del diseño; por consiguiente, actualmente en el ámbito docente, el proceso de enseñanza aprendizaje de técnicas o herramientas para el conocimiento de usuarios es un tópico importante y el principal contenido de algunas Unidades de Enseñanza Aprendizaje (UEA) o materias en la licenciatura en diseño en la Universidad Autónoma Metropolitana, Unidad Cuajimalpa (UAM Cuajimalpa).

Los contenidos de dicha enseñanza en la UAM Cuajimalpa actualmente comprenden herramientas tomadas de áreas como la Etnografía, Factores Humanos, Usabilidad, y el Diseño Participativo. Es indudable que dichas herramientas presentan una gran utilidad e importancia. Podemos decir que una de sus características más relevantes, desde la perspectiva humana, es que presentan lo que se puede denominar un enfoque "hacia afuera", es decir exterior al estudiante, ya que se apoyan principalmente en la observación, el cuestionamiento, y la indagación del grupo de usuarios al que se dirigirá el proyecto. El "enfoque hacia afuera" es importante porque es útil para conocer algunos aspectos o características del usuario que son externas a él y en gran medida fácilmente identificables por medio de técnicas como la observación y entrevistas principalmente.

Por otro lado, se considera que también existe una oportunidad en lo que aquí se ha denominado "un enfoque hacia adentro" ya que permitiría contar con una manera de comenzar a reconocer otras dimensiones de las personas, cuya naturaleza es interna y en gran medida difícil de identificar al observar o cuestionar, como lo es la dimensión emocional ya que es muy común que un individuo no sea totalmente consciente de las emociones que vive en 
un momento dado. El enfoque "hacia adentro" que aquí se propone consiste en fomentar el desarrollo de la habilidad de la empatía, con base en la introspección y la experiencia personal del alumno o futuro diseñador frente al usuario y su contexto de manera que le permita al estudiante reconocer al otro a partir de identificar manifestaciones y necesidades humanas del ámbito emocional que tienen en común (como personas que son), de este modo se busca introducir al estudiante al conocimiento de usuarios en aspectos humanos de naturaleza distinta a los generalmente abordados hasta hoy; se trata de un camino que implica iniciar con un proceso interno del individuo, que tiene como fundamento la búsqueda por dar respuesta al cuestionamiento: ¿qué reconozco en mí que también puedo reconocer en los demás?

De este modo el objetivo que persigue este trabajo consiste en fomentar la habilidad de la empatía en los futuros diseñadores a partir de la introspección, como un primer paso para concebir estrategias que conduzcan al futuro diseñador a conocer a los usuarios principalmente en su dimensión emocional, que es en gran medida interna y subjetiva.

El desarrollo del presente trabajo comenzará presentando la definición de empatía desde varias disciplinas, principalmente desde la psicología y se señalará por qué la introspección con un "enfoque desde dentro" ayuda a iniciar el camino para entender al otro, posteriormente se presentará la definición de empatía desde el diseño, algunos ejemplos de intentos de búsqueda por enseñar a los alumnos a ser empáticos "desde dentro"; y como último punto se expondrá una experiencia docente que buscó estimular la empatía con base en la introspección durante el proceso de enseñanza aprendizaje del diseño, esta fue una primera experiencia con el fin de dotar de herramientas al alumno para el conocimiento del usuario, de manera que se reportará la utilidad e importancia de nuestra propuesta para la formación del alumno o futuro diseñador a partir de un ejercicio interno por parte del estudiante. 


\section{Definición de empatía desde otras disciplinas}

El estudio de la empatía es una habilidad humana relevante, aquí se aborda su definición desde diversas áreas del saber, con el fin de reflexionar y visualizar nuevos caminos para su integración al diseño.

Desde la sociología, la empatía se ha definido como:

\section{(...) habilidad tanto cognitiva como emocional del individuo, en la cual éste es capaz de comprender la situación emocional del otro, a otra cultura, a otro modo de vida, otra creencia, u otro momento histórico. Significa poder entender sus necesidades, deseos o acciones (Rifkin, 2010, 22).}

Desde el ámbito de la medicina, la empatía es una característica esperable en el médico, que le permita desempeñar roles significativos y le ayude a mejorar su relación con el paciente. Paralelamente se espera que la empatía del médico favorezca un adecuado diagnóstico, así como la mejora a la adherencia al tratamiento y a los resultados del mismo (Cooper, 2004). En el área de enfermería igualmente se hace mención de la empatía para el trato a pacientes.

\section{Definición de empatía desde la psicología}

La disciplina que más ha abordado el tema y para la cual la empatía ha sido un importante concepto y temática de muchas reflexiones y publicaciones es la psicología.

Rifkin (2010), en su obra La civilización empática, expone que la importancia que se ha dado en la psicología y en otras ciencias a la empatía, ha ido creciendo constantemente hasta que ha pasado a ocupar un lugar central, mismo que refleja la importancia que tiene para todo ser humano; ya que, plantea el autor, es parte importante de nuestra naturaleza el sentir empatía, de hecho, señala, los individuos estamos "cableados" para ello, la empatía es la base material que nos permite ser seres sociales. 
Diversos autores que han publicado trabajos sobre la historia de la psicología (Hakansson, 2003; Nilsson, 2003; Jahoda, 2005) señalan el origen de la empatía en 1873 en el área de historia del arte, específicamente en el hecho de que el filósofo y poeta alemán Vischer utilizó la palabra einfühlung término alemán para el vocablo sentimiento y que describía un proceso en el cual una mujer proyecta su personalidad sobre un objeto y en algún sentido se fusiona con éste. El psicólogo Theodor Lipps utilizó dicho término para explicar la experiencia estética, y destinó el término a la descripción de la experiencia de las personas y al conocimiento de los estados mentales de los individuos (Nilsson, 2003).

Lipps propuso que las personas conocen y responden al otro a través de einfühlung el cual es generado por proyección e imitación, especialmente imitación de afecto, por ejemplo, sonreír cuando se observa que alguien más sonríe. Este trabajo es importante porque ilustra directamente el factor afectivo de la empatía.

Titchener fue quien trasladó el término alemán hacia el vocablo inglés empathy del griego em - hacia y pathos - pasión, sentimiento. En 1915 escribió que la empatía es importante en la imaginación ya que los individuos tenemos una tendencia natural a sentirnos a nosotros mismos en aquello que percibimos o imaginamos y esta tendencia a sentirse uno mismo hacia una situación es llamada empatía, que es sentir junto con otros.

Esta referencia del "sentirse uno mismo en aquello que percibimos o imaginamos" en la definición de empatía de Titchener hace alusión a la introspección ya claramente, y por lo tanto es la base sobre la que es posible que un individuo entienda al otro, al prójimo.

Otro autor que es relevante mencionar es el psicólogo humanista Rogers (1961), quien entiende la empatía como la comprensión íntima del otro, del mundo interno del otro, tal como éste lo vive, aunque no sepa con claridad cómo lo vive. Es la percepción del otro en uno, como alguien que no soy yo, pero que me resulta tan familiar como lo soy para mí mismo, de allí que se pueda afirmar que si me conozco a mí mismo puedo tener una idea mucho más clara del prójimo. 
La comprensión empática es un concepto clave de la psicología humanista de Rogers (1961), es una de las tres actitudes básicas que señala debe de tener un terapeuta para el logro de un proceso exitoso, las cuales son: congruencia o integridad, aceptación positiva incondicional y comprensión empática o simplemente empatía.

A través de las técnicas de la psicología humanista se invita al psicólogo a involucrarse emocionalmente con el paciente, con el fin de establecer entre ambos un verdadero vínculo de encuentro.

El modelo humanista, respecto al ser humano, expresa entre otras características que cada persona es un sistema configurado, que considera la singularidad individual, que es lo particular, y también lo general, dado que pertenece a la condición de seres humanos. Lo anterior permite inferir que conocerse y entenderse uno mismo es conocer y entender de alguna manera a los semejantes (Villegas, 1986).

Las diferentes visiones en torno a la empatía recaen en el individuo, y eso justifica el desarrollo de la introspección del individuo como punto de partida de la empatía.

\section{La introspección como punto de partida de la empatía}

Con base en la idea central de que la introspección permite que un individuo entienda al otro desde la realidad del ser humano; en éste apartado se amplía dicho planteamiento desde dos estudios del ámbito de la psicología uno referente al componente fisiológico que hace posible la empatía del ser humano a partir de su propia constitución biológica, las neuronas denominadas "espejo" y el otro relacionado con el desarrollo vital de una persona, el desarrollo de la consciencia de uno mismo. 


\section{Las neuronas espejo y la empatía}

La explicación a nivel fisiológico de la empatía es a través de las neuronas denominadas "espejo" descubiertas en 1995 por Giacomo Rizzolatti, se trata de un tipo de neuronas que se activan durante el proceso de observación atenta a otros seres humanos, dicha activación ocurre en la misma cantidad y región cerebral de las del sujeto observado y permiten a los humanos y a otras especies de animales reaccionar ante el otro, como si la conducta y el sentir de esos otros fueran los suyos. (Redolar, 2013; Mukamel, 2010).

Las neuronas espejo hacen posible aprender imitando: sonreír, caminar, bailar, aunque también al mismo tiempo nos conducen a sentir pena cuando alguien llora, o a experimentar alegría compartida. Cuando una persona realiza acciones en contextos significativos, dichas acciones van acompañadas de la captación de las propias intenciones que motivan a hacerlas. Se conforman sistemas neuronales que articulan la propia acción asociada a la intención o propósito que la activa. La intención queda vinculada a acciones específicas que le dan expresión, y cada acción evoca las intenciones asociadas. Formadas estas asambleas neuronales en un sujeto, el observador reacciona a la acción equivalente, evocando la intención con ella asociada. El sujeto, así, puede atribuir a otro la intención que tendría tal acción si la realizase él mismo (García, 2008).

La lectura que una persona hace de las intenciones del otro, es en gran medida, atribución desde las propias intenciones; conocer la importancia y utilidad de estas neuronas explica el hecho de que las personas afectadas de autismo al sufrir precisamente de alteraciones en la actividad de sus neuronas espejo, presenten por lo tanto incapacidad para deducir las intenciones ajenas y vivenciar la empatía (García, 2008).

Los seres humanos somos criaturas sociales y nuestra supervivencia depende de entender las intenciones y emociones que traducen las conductas manifiestas de los demás. Las neuronas espejo permiten entender la mente de nuestros semejantes, y no a través de razonamiento conceptual, sino directamente, sintiendo y no pensando (Rizzolatti, Fogassi \& Gallese, 2001). 


\section{La conciencia de uno mismo}

La capacidad empática de una persona, en primer lugar depende en gran medida del desarrollo de la conciencia del yo, de su condición de ser humano. De relevancia central es la relación entre individualización y empatía, cuanto más individualizado está el yo, más intensa es la sensación de que nuestra existencia es única, finita, mortal, y más profunda es la conciencia de nuestra soledad existencial y la de los muchos y difíciles retos que hemos de afrontar para poder sobrevivir y prosperar. Lo anterior es significativo, porque son precisamente las sensaciones de sobrevivencia y prosperidad las que permiten sentir empatía con los demás (Rifkin, 2010).

Al mismo tiempo, la importancia del desarrollo de la conciencia de uno mismo es fundamental para entender al prójimo. Erikson (1983) señala que parte central del proceso de desarrollo emocional del niño es precisamente la conciencia de sus propios estados emocionales, habilidades y potencial para la acción, fenómeno llamado "conciencia de uno mismo" (Self Awarness).

Así, desde que un ser humano se encuentra en la etapa de la infancia, la creciente consciencia y habilidad para reconocer sus propios estados emocionales, guía su empatía o su habilidad para apreciar emociones, sentimientos o percepciones de los otros (Erikson, 1983). Con base en lo anterior, cobra mayor relevancia presentar el proceso de la empatía a través del modelo denominado rueda de la empatía (Wheel of empathy) que se presenta en la Figura 1. El planteamiento que lo sustenta es que la empatía surge del sentir personal de una experiencia determinada vivida por una persona. La rueda de la empatía señala cuatro aspectos básicos que la integran y subrayan la importancia de la introspección del "sujeto empático". 
SOBRE LA EMPATÍA Y LA INTROSPECCIÓN

EMOCIONAL EN LOS DISEÑADORES

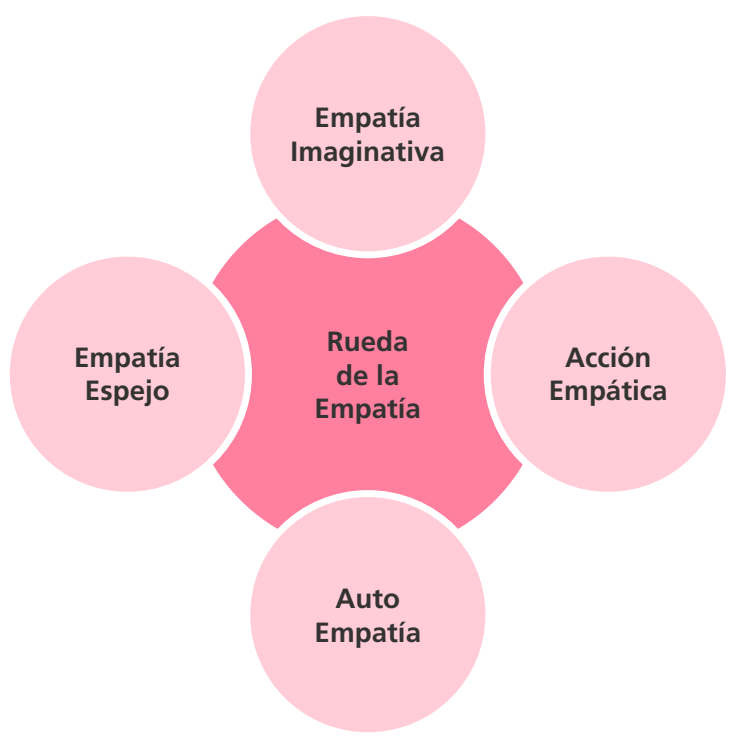

Figura 1. Modelo denominado Wheel of Empathy que señala cuatro aspectos básicos que integran el proceso de la empatía [Elaboración y traducción por la autora]. Fuente: *Center for Building a Culture of Empathy http://cultureofempathy.com/references/definitions.htm

Los cuatro aspectos básicos de dicho modelo son:

1 Auto empatía (self empathy). Traducir los juicios internos hacia sentimientos y necesidades.

2 Empatía espejo (mirrored empathy). Desarrollar la empatía emocional con los demás a través de las neuronas espejo.

3 Empatía imaginativa (imaginative empathy). Reconocer el ser distinto del individuo y al mismo tiempo reconocerse como los demás. Esto permite desarrollar la habilidad de toma de roles y considerar las perspectivas de otros individuos.

4 Acción empática (empathy action). Resolver el rompecabezas de organizar nuestra experiencia compartida, buscando modos creativos de entender a los demás.

Con base en este modelo, es posible mencionar que el entendimiento empático va más allá de factores cognitivos e incluye la dimensión afectiva: cuando empatizamos, el individuo se relaciona con el otro entendiendo la situación y sobre todo, con base en que ciertas experiencias son significativas para él al igual que para los otros, de este modo se trata de una relación que involucra una conexión emocional individual importante con el otro. 


\section{Definición de empatía desde el diseño}

De acuerdo con Battarbee y Koskinen (2005) el adjetivo "empático" en relación al diseño fue introducido en la década de los 90s, cuando algunas compañías se empezaron a dar cuenta que solamente escuchar las respuestas de los clientes a los cuestionarios que se les aplicaban no era suficiente para el desarrollo exitoso de productos industriales (Leonard \& Rayport, 1997; Sanders \& Dandavate, 1999).

Esto dirigió la vista de los diseñadores, en estrecha colaboración con dichas compañías, hacia la necesidad de ser más sensibles a los usuarios, a ser capaces de entenderlos, entender su situación, sentimientos, a ser empáticos con ellos.

Así, el término "empatía" apareció en el horizonte de la disciplina; Battarbee (2004) describe el proceso de diseñar con empatía como "dejar la oficina (de diseño) y empezar a sumergirse en las vidas, ambientes, actitudes, experiencias y sueños de los futuros usuarios, internalizando sus requerimientos"(25). La empatía, dicen Mattelmäki y Battarbee (2002) es la base del cambio a un proceso de diseño basado en consideraciones de un diseño racional y práctico a uno de experiencias y contextos personales del usuario.

En el libro Empathic Design (Koskinen et al., 2003), distintos profesionales del diseño discuten y reflexionan sobre el rol de la empatía en el diseño. Todos los autores coinciden que la empatía es una cualidad necesaria en el proceso de diseño, con el fin de desarrollar productos que realmente conozcan las necesidades de las personas o usuarios. Esta conclusión es interesante porque desde hace décadas se argumenta que se diseña para las personas, cuando la realidad indica que hay una desconexión entre el diseñador y los usuarios (ver Ortíz-Nicolás, 2016).

Otros describen al diseño empático como una proyección imaginativa hacia otra persona y situación (Koskinen \& Battarbee, 2003), o un tipo particular de imaginación (Fulton Suri, 2003). Los términos imaginación y proyección implican que ser empáticos requiere de actividades donde los diseñadores imaginen qué podría gustarles a ellos desde la posición de un 
usuario. Es relevante comentar que esta postura sugiere la importancia para el diseñador de entender lo que significa ser humano desde la autorreflexión para imaginar cómo es la vida de las personas para las que va a diseñar considerando que también son seres humanos.

Además de ser una cualidad del proceso de diseño, la empatía ha sido también descrita en el área como una habilidad que tienen las personas (McDonagh, 2006), por lo consiguiente es lógico deducir que como toda habilidad humana, consta de distintas destrezas, los individuos diferirán en el grado de habilidad que presenten para cada una de ellas. Así, McDonagh (2006) define la empatía como "la habilidad intuitiva para identificarse con los pensamientos e ideas de otras personas, sentimientos, motivaciones modelos emocionales y mentales, valores, prioridades, preferencias, y sus conflictos internos o personales"(3).

De este modo existe la posibilidad de guiar al futuro diseñador a partir de la introspección, al autoconocimiento y conciencia de su mundo empático, a conectar y ser sensible con el otro. La dimensión emocional humana puede ser la conexión directa entre diseñador y usuario, de tal forma que le permita al alumno sentir un compromiso personal y directo, y por lo tanto tener la disposición necesaria para conocer y atender mediante el diseño las necesidades del otro.

Ello es posible en el horizonte empático de los diseñadores, independientemente de si él o ella es naturalmente empático, al ser ésta una habilidad humana puede ser paulatinamente aprendida y extendida mediante entrenamiento y experiencia en la práctica de su desarrollo, sobre todo siendo abordada en primer lugar como un acto de introspección (Cooper, 2004).

\section{Enseñanza de la empatía en el diseño y sus enfoques}

En esta sección se presentan algunos ejemplos puntuales del ámbito docente, con el fin de conocer concretamente cómo se da el proceso de enseñanza - aprendizaje de la empatía dentro de la disciplina del diseño, para lo cual se realizó una búsqueda general sin ser exhaustivos, de casos específicos relacionadas al objetivo de este artículo. 
Como resultado de dicha búsqueda es posible mencionar que el número de textos existentes del tema es limitado; el trabajo de Cote y Gagnon (2014), tuvo como objetivo principal enseñar a los estudiantes a ser empáticos, sin embargo, dicho entrenamiento se realizó desde un enfoque "hacia afuera" que como hemos mencionado, considera el conocimiento del usuario a través de una caracterización de su perfil y poco más, obtenido primordialmente mediante la escucha y observación del usuario.

Por otra parte, efectivamente se encontraron ejemplos de trabajos con un enfoque "hacia dentro", es decir, basados en el conocimiento y conciencia del propio mundo emocional interior del futuro profesional del diseño, de manera que su autoconocimiento le guiara y permitiera reconocerse como un ser humano sensible al usuario y por lo tanto buscar conectar en una condición similar y empática con los usuarios de sus propuestas de diseño. Es este segundo tipo de trabajos en el que se hará énfasis.

\section{Modelo de Merlijn y Froukje (2009)}

Paralelamente a la búsqueda por definir lo que es la empatía dentro del diseño, algunos de los autores mencionados sugieren herramientas y técnicas, concebidas para guiar y ayudar al profesional del diseño a integrar la empatía en sus proyectos. Se trata por ello de herramientas o técnicas que han sido propuestas desde el mundo profesional del diseño (despachos o investigación) y cuya aplicación permanece principalmente en él, es de importancia comentarlas, a reserva de presentar algunos ejemplos de experiencias particulares al ámbito docente.

Merlijn y Froukje (2009) han clasificado en tres tipos principales a las herramientas o técnicas para conocer al usuario:

a Herramientas para el contacto directo entre diseñadores y usuarios.

b Técnicas para comunicar hallazgos de grupos de usuarios a equipos de diseño.

c Técnicas para evocar las propias experiencias del diseñador en un ámbito relevante para el usuario. 
De estos tres grupos de herramientas o técnicas, las señaladas en el inciso $\mathrm{C}$ que buscan evocar las propias experiencias del diseñador en un ámbito relevante para el usuario, las abordaremos con más detalle, porque apuntan a la introspección del diseñador, que es el tema central de este trabajo.

Merlijn y Froukje (2009) conducen al diseñador a simular la condición del usuario a través de tres versiones de "juegos de roles", denominadas como "experience prototyping", "body storming" e "informance" (Merlijn \& Froukje, 2009).

El desarrollo de la técnica de "juegos de roles" (Laurel, 2003) se llevan a cabo mediante métodos teatrales y atmósferas ambientales sugestivas (Keller \& Stappers, 2001). El diseñador toma la perspectiva del usuario y actúa. Buchenau y Fulton Suri (2000) refieren que esta técnica permite llevar a cabo el proceso de explorar nuevos conceptos de producto o interacciones mediante simulaciones, que es un tipo de representación por cualquier medio, diseñada para entender, explorar o comunicar qué podría gustar, o enganchar al usuario. El diseñador al enfocarse a situaciones particulares es capaz de obtener nuevas ideas (Buchenau \& Fulton-Suri, 2000).

Con base en lo expuesto, es posible decir que la empatía ha sido y es un tema de interés en el ámbito del diseño y del cual ya se han dicho distintas cosas y aún quedan por decir, por ejemplo señalar propuestas que aporten mecanismos psicológicos para conducir al presente y futuro diseñador a crear conciencia, entendimientos emocionales, relaciones, y conexiones, que lo guíen a internalizar la experiencia de otra persona, a ser realmente empático con ella e ir más allá de esa observación del usuario, siendo que es igualmente un individuo, un ser humano, una persona, un usuario.

Por otro lado, es relevante retomar el planteamiento de Merlijn y Froukje (2009) referente a que la empatía es una cualidad admitida del proceso de diseño y buscada por el diseñador pero cuya definición de lo que es exactamente queda aún en el campo de la intuición, lo que limita su estudio e implementación. 


\section{Modelo de Tomberg y Laanpere (2014)}

El trabajo de Tomberg y Laanpere (2014) implementa un modelo empático en el programa de Human Computer Interaction ( $\mathrm{HCl}$ ), que se inspira en Reik (1949). El modelo guía al alumno a ser empático a través de cuatro fases:

a Identificación: enfocar su propia atención en el otro y permitiendo así mismo llegar a ser absorbido en contemplación de esa persona

b Incorporación: hacer que la experiencia del otro sea internalizada por sí mismo

c Reverberación: reflejarse, vivir la experiencia de otros a través de atender a las propias asociaciones personales cognitivas y afectivas de esa experiencia.

d Desapego: regresar de la relación interior fusionada a una posición de identidad separada, que permite una respuesta que refleja el entendimiento de otros tanto como separado de ellos.

En el análisis de dicha experiencia docente, los autores mencionan haber obtenido buenos resultados, sobre todo porque el proyecto sobre el que trabajaron iba dirigido a personas con determinadas discapacidades principalmente sensoriales (ceguera y sordera), por lo cual la empatía fue un elemento central para que el alumno fuera capaz de entender a los usuarios con características y necesidades tan específicas en dicho proyecto.

Con estas propuestas, se plantea y se guía a un importante cambio de enfoque en el conocimiento de usuario, de aquél que había de proyectar con base en las características descriptivas de un perfil de usuario, como se había venido haciendo tradicionalmente; a uno que busca visualizar aspectos que pertenecen principalmente al ámbito del comportamiento y experiencia de los individuos.

Siendo la empatía una característica y habilidad tan relevante, compleja y rica a nivel humano, es capaz de aportar mucho más a la disciplina del diseño y por lo tanto a los diseñadores presentes y futuros. 


\section{Estudio}

En la UAM Cuajimalpa, se considera importante que los alumnos conozcan al usuario a partir de la empatía basada en la introspección. Existe una primera experiencia, que aquí se expondrá, la cual busca desarrollar la empatía en los alumnos, esto particularmente en la impartición del tema "diseño y emociones" dentro de la materia "laboratorio de diseño integral III" del plan de estudios de la licenciatura en diseño de la UAM Cuajimalpa. Dicha materia pertenece al segundo año y su objetivo es el estudio y manejo por parte del alumno de la dimensión expresiva del diseño.

Teniendo como marco el planteamiento de Erikson (1983) y el modelo denominado "wheel of empathy" ambos ya descritos en este trabajo, se estructuró una actividad cuyo objetivo principal fue conducir al alumno a reflexionar en torno a la experiencia emocional que cada uno vivía, de manera que los llevara a la investigación e identificación, y finalmente a la comprensión de sus propias emociones y sentimientos. Se escogieron las emociones porque son cualidades intrínsecamente humanas, principalmente subjetivas e internas al individuo y en muchos aspectos no fácilmente reconocibles al ser observadas o cuestionadas por ser en muchos casos el individuo no totalmente consciente de ellas. La actividad se concibió como una manera de comenzar a introducir la empatía desde la introspección en dicho curso.

\section{Participantes}

Veintiún participantes asistieron al ejercicio, todos estudiantes de la Licenciatura en Diseño de la Universidad Autónoma Metropolitana unidad Cuajimalpa; doce hombres y nueve mujeres, con un promedio de edad de veintiún años de edad. 
SOBRE LA EMPATÍA Y LA INTROSPECCIÓN

EMOCIONAL EN LOS DISEÑADORES

\section{Procedimiento}

La actividad, consistió en conducir al alumno a hacer un fuerte hincapié en la reflexión y exploración de su experiencia emocional personal; para ello se siguieron cuatro etapas:

a Elección de producto. Se les solicitó a cada uno de ellos la elección de un producto que a nivel personal les fuera emocionalmente significativo.

b Creación de un diario de uso. Una vez elegido el producto se les pidió la creación de un diario para reportar la experiencia afectiva (emociones y sentimientos) que vivían al interactuar y/o hacer uso del objeto elegido. Para la creación del diario se les proporcionó una guía establecida por la autora de este texto y docente del grupo (Figura 2). Dicha guía les planteó tres preguntas con el objetivo de guiarlos a ser conscientes de la experiencia emocional vivida durante su interacción con el objeto elegido: ¿en qué momentos usé el objeto? ¿Cómo lo usé?, ¿Cómo me sentí al usarlo?.

c Análisis sistemático del diario de uso. Una vez que se respondieron los diarios, se realizó el análisis individual y grupal del mismo, para lo cual se proporcionó un formato (Figura 3) para guiarlos en la traducción de sus juicios internos hacia emociones, sentimientos y necesidades. Esta fase fue determinada con base en el punto 1, auto empatía del modelo rueda de la empatía.

d Acción Empática. Como última fase se les condujo a la reflexión en relación al usuario meta del producto, los estudiantes lo determinaban de acuerdo a su experiencia. A partir de considerar su propio sentir, se retomó la interrogante: ¿Qué reconozco en mí que también puedo reconocer en otros usuarios de este producto? Se trató de un trabajo de reflexión, de comparación, pero principalmente, de reconocimiento de la naturaleza humana, de constantes humanas emotivas y afectivas innegables, muchas de ellas muy evidentes. 


\begin{tabular}{|c|c|}
\hline \multicolumn{2}{|l|}{ Diario de uso } \\
\hline \multicolumn{2}{|l|}{ Nombre } \\
\hline \multicolumn{2}{|l|}{ Fecha } \\
\hline \multicolumn{2}{|l|}{ Objeto elegido } \\
\hline \multicolumn{2}{|l|}{ Día 1} \\
\hline Cuestionamiento & Descripción narrativa \\
\hline \multicolumn{2}{|l|}{ ¿En qué momentos lo usé? } \\
\hline \multicolumn{2}{|l|}{ ¿Cómo lo usé? } \\
\hline \multicolumn{2}{|l|}{ ¿Cómo me sentí al usarlo? } \\
\hline \multicolumn{2}{|l|}{ Día 2} \\
\hline Cuestionamiento & Descripción narrativa \\
\hline \multicolumn{2}{|l|}{ ¿En qué momentos lo usé? } \\
\hline \multicolumn{2}{|l|}{ ¿Cómo lo usé? } \\
\hline ¿Cómo me sentí al usarlo? & \\
\hline
\end{tabular}

Figura 2. Tabla guía que integró el formato del diario de uso proporcionado a los alumnos para el desarrollo del ejercicio.

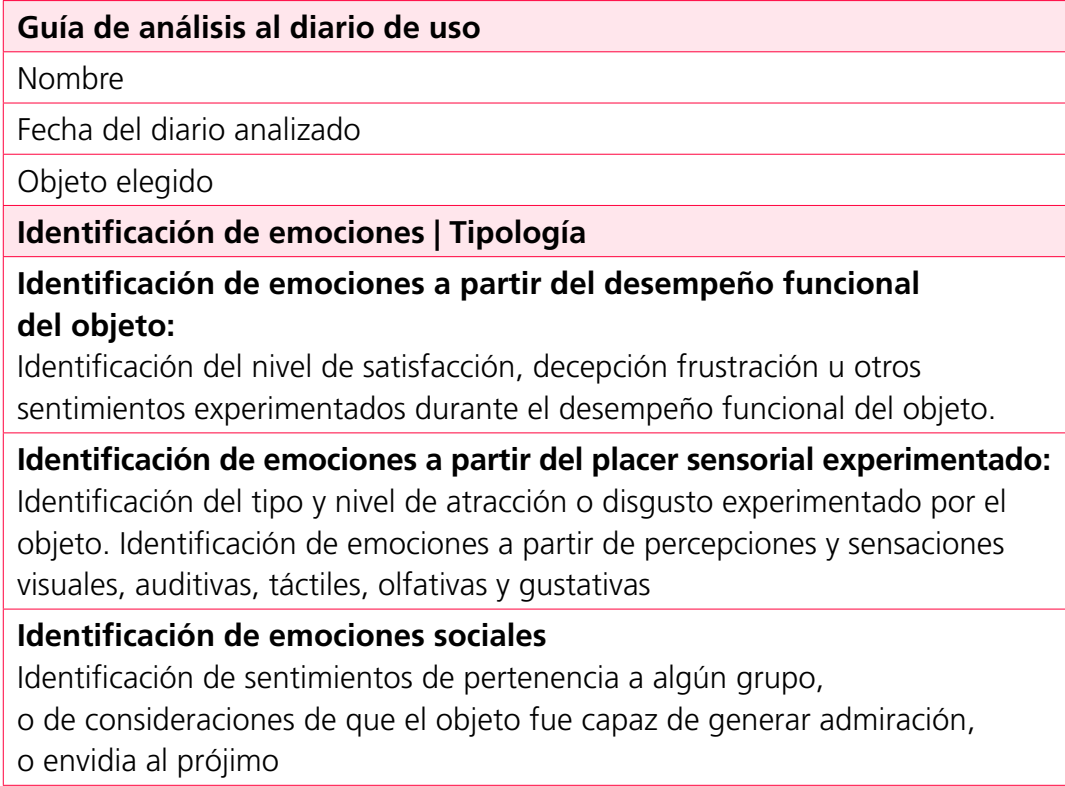

Figura 2. Formato guía para el análisis de los diarios de uso. 
El formato guía utilizado fue desarrollado con base en tres de las cinco categorías de respuestas emocionales identificadas y propuestas por Desmet (2002), los cuales se reportan a continuación:

1 La identificación de emociones a partir del desempeño funcional del objeto es decir a la identificación del nivel de satisfacción, decepción, frustración u otros sentimientos experimentados durante el desempeño funcional del objeto.

2 La identificación de emociones a partir del placer sensorial, es decir la identificación del tipo y nivel de atracción o disgusto experimentado por el objeto y a la identificación de emociones a partir de percepciones y sensaciones visuales, auditivas, táctiles, olfativas y gustativas vividas.

3 La identificación de emociones sociales, es decir, la identificación de sentimientos de pertenencia a algún grupo, o consideraciones de que el objeto fuera capaz de generar admiración, o envidia al prójimo.

\section{Resultados}

Se obtuvieron 21 diarios de uso. Los objetos elegidos fueron muy diversos:

Teléfonos celulares (2), iPod (2), cepillo de dientes (1), almohada (1), mochilas (2), prendas de vestir distintas (3), bicicleta (1), gorra (1), pluma (1), ordenador portátil (1), termo (1), taza (1), tenedor (1), cama (1), reloj de pulso (1), automóvil (1).

Los alumnos fueron capaces de identificar que los objetos generan distintas emociones y sentimientos con los productos.

Al haberse tratado de un trabajo de reflexión, de comparación, y principalmente de reconocimiento de la naturaleza humana, los alumnos fueron capaces de entender que su propio sentir tiene muchas similitudes con el de los demás, al tratarse de constantes humanas emotivas y afectivas innegables. Estas parten de necesidades innatas derivadas de requerimientos afectivos básicos y de necesidades secundarias también muy importantes, ya que 
SOBRE LA EMPATÍA Y LA INTROSPECCIÓN

EMOCIONAL EN LOS DISEÑADORES

responden a la naturaleza humana misma. Entre dichos requerimientos podemos mencionar por ejemplo la identificación de la búsqueda innata de aceptación de todo individuo por el prójimo, la identificación de la búsqueda de sentir seguridad y confort, la necesidad de dar y recibir amor, cariño, cuidados, y lo significativo de los seres queridos para todo individuo.

Con base en lo anterior, retomar la pregunta ¿Qué reconozco en mí que también puedo reconocer en otros usuarios de este producto? Les hizo comprender a los alumnos que ellos mismos son capaces, en un momento dado, de ser inicialmente el referente humano en muchos sentidos, en especial para entender aspectos tan complejos y subjetivos como las emociones y necesidades afectivas específicas. Si bien este es un paso inicial, quizá puede ser un medio-guía para indagar posteriormente con mayor profundidad la dimensión emocional en los usuarios.

El hecho de que la introspección representa un primer paso está fundamentado en el concepto de Rogers (1961) de lo que es la empatía, es un primer paso en la comprensión íntima del otro, del mundo interno del otro, tal como éste lo vive, aunque no sepa aún con claridad cómo lo vive. Es la percepción del otro en uno, como alguien que no soy yo, pero que me resulta tan familiar como lo soy para mí mismo.

El ejercicio desarrollado por los alumnos los condujo a reflexionar que el usuario no es un ser distinto del todo a ellos, por lo cual es de gran utilidad el autoconocimiento que en muchos sentidos es semejante a los demás.

\section{Discusión}

Las reflexiones finales del trabajo desarrollado, se exponen aquí desde tres perspectivas que permiten abordar los diversos aspectos tratados: reflexiones sobre la adaptación o congruencia de las técnicas empleadas con los alumnos que participaron en el ejercicio para la formación inicial en la empatía, la utilidad y naturaleza de la herramienta docente frente a las características de la habilidad empática en la que se quiere formar, y los aspectos pen- 
dientes a reflexionar y desarrollar para la formación en la empatía en la disciplina del diseño. Sobre las técnicas empleadas en el proceso de enseñanza aprendizaje con los alumnos, como análisis de esta primera experiencia, se puede decir que hubo una buena participación de ellos en general, sin embargo, visualizar y expresar su propio mundo emocional fue una tarea que realizaron con dificultad, debido a sus inhibiciones y a la existencia de algunos prejuicios, como lo son por ejemplo aquellos derivados de los modelos de comportamiento de género en el que el hombre reprime ciertas emociones por no ser consideradas masculinas.

Es importante destacar que el análisis de las emociones y sentimientos registrados en los diarios desarrollados por los alumnos, así como la guía proporcionada para su análisis sistemático, fueron fundamentales para la discusión plenaria e intercambio de ideas y experiencias, lo cual permitió guiar a los estudiantes a la identificación y descubrimiento del conjunto de sentimientos, emociones, y características inherentes a la naturaleza humana, que son una constante innegable para todo individuo aunque existan diferencias en el modo en que se vivan y en la intensidad experimentada.

Otro aspecto importante que a nivel grupal se buscó que fuera comprendido claramente, es que la empatía parte del ser que la busca o experimenta, es decir, un diseñador es empático con otra persona (usuario) cuando así lo procura, por lo que la empatía está influida por la voluntad, los conocimientos, experiencia, y habilidades que adquiera el diseñador. Por esta razón ejercitar el enfoque introspectivo para llegar a ser empático es fundamental para la práctica de un diseño que se busque esté en verdadera congruencia con el usuario. Cabe mencionar que en cierta medida, ésta sería la gran limitación de la empatía, que enfoques como el pensamiento de diseño la tiendan a ignorar.

Como herramienta docente esta experiencia, representa una primera aproximación para explorar la utilidad de la introspección del alumno; un modo que permite comenzar a fomentar la habilidad de la empatía del estudiante con los usuarios actuales o potenciales de un producto o servicio, principalmente para conducirlos al aprendizaje de temas tan 
complejos como la identificación de las emociones y los sentimientos humanos en relación a la interacción con objetos o productos; es claro que se trata de un tema que no es tan fácil de abordar debido a su naturaleza subjetiva, por lo cual en este caso inicial, el enfoque se basó en un principio del factor humano general: las personas invariablemente experimentan emociones y sentimientos ante un estímulo, un producto o servicio (Bedolla, 2002; Desmet, 2002; Norman, 2004).

La introspección como medio para formar la empatía en los estudiantes de diseño, requiere una reflexión más profunda respecto a los siguientes pasos a seguir para lograrlo. Desarrollar la habilidad de la empatía en la disciplina del diseño demanda una estrategia específica en congruencia con el quehacer y la naturaleza de la disciplina, del mismo modo que lo han hecho otras como medicina y enfermería, cuyo interés en la empatía ha sido constante, por lo cual han desarrollado métodos y estrategias (Cooper, 2004), que responden claramente a sus necesidades.

En este trabajo se reconoce que es necesario seguir explorando y reflexionando dicho camino formativo en la empatía para llegar a concebirlo completamente. Encontrar coincidencias y semejanzas del sentir y vivir entre dos individuos, es un paso inicial hacia el camino que llevará a conocer al otro en su propia naturaleza y circunstancia. Es claro que existe la necesidad de continuar con el proceso para desarrollar la empatía ampliamente, lo que implica que las personas estén preparadas para empatizar no solo con aquellos que presenten semejanzas sino también con aquellos que son distintos a él/ella.

Lograr empatizar con personas distintas a uno mismo requiere otra fase de la empatía que vaya más allá de la introspección; ello está señalado en el tercer aspecto básico del proceso de la empatía propuesto en la "rueda de la empatía" (Wheel of empathy) que refiere a la "empatía imaginativa" (imaginative empathy). Esta busca reconocer al individuo empático como distinto del que ha observado, con el fin de tomar diferentes roles y considerar las perspectivas de otros individuos. La empatía imaginativa está considerada también en el trabajo de Tomberg y Laanpere (2014), ellos guían al alumno a ser empático a través de 
cuatro fases, la última es denominada "desapego" y propone establecer una posición de "identidad separada", que permite una respuesta que refleja el entendimiento de otros como separado de uno mismo. Este nivel de la empatía resulta muy útil con personas muy distintas a los diseñadores, motivo por el que ha sido utilizado en proyectos orientados a personas con discapacidad sensorial (ceguera y sordera), de este modo la empatía "con desapego" es un medio para que estudiantes de diseño reflexionen en lo que implica tener una discapacidad y generen soluciones a partir de dicha reflexión. Es realmente difícil conocer cómo es ser ciego sin serlo, pues la discapacidad simulada por el proyectista en tal caso es temporal y la de las personas ciegas no.

Con base en lo anterior, la siguiente fase que se propone aquí con el fin de continuar con el proceso para conducir al alumno a desarrollar la empatía completamente, se refiere a guiarlos hacia el fomento e integración de un componente del proceso humano de la empatía denominado "componente cognitivo". El componente cognitivo de acuerdo con Morse (1992) es el que se refiere a la habilidad intelectual para entender la perspectiva del otro; componente que es posterior a la disposición empática inicial (componente moral), y a la habilidad para percibir subjetivamente los sentimientos del prójimo (componente emotivo), pero que es previo a la capacidad del individuo para comunicar su preocupación y comprensión empática.

La fase posterior a la introspección en la formación de la empatía, requiere fomentar el ser sensible con el otro, a pesar de tratarse de individuos completamente distintos a él con los que es necesario empatizar y ser sensible a su propia problemática aunque sea muy lejana y ajena; ello estaría en congruencia con lo que señala Bloom (2016) al mencionar la importancia y utilidad actual de la consideración y aplicación amplia de la empatía en todos los sectores y niveles para lograr tener una influencia humana significativa más amplia, lo cual señala Bloom incluye a nivel macro, a aquellos cuya tarea es el establecimiento de políticas públicas que sean socialmente relevantes. 
Este ejercicio exploró la dimensión emocional, la afectividad del ser humano, sin embargo hay otras dimensiones que son también relevantes e intrínsecamente humanas, como la corporeidad, imaginación, etc. Y por lo tanto, esas son otras áreas de oportunidad para seguir desarrollando la autorreflexión y para madurar la habilidad empática de los diseñadores.

Por otro lado, es importante señalar que en esta experiencia en particular se recurrió a herramientas específicas como son los diarios y el uso de un producto relevante para los estudiantes, si bien este enfoque dio resultados, quizá hayan otros medios que también sean útiles para estimular la reflexión e introspección en el alumno, por lo tanto es posible continuar la búsqueda y desarrollo de otras herramientas adicionales que también puedan ser idóneas para guiar en la experiencia didáctica auto reflexiva.

La empatía tiene todavía mucho que aportar en el ámbito del diseño, por lo cual es necesario generar propuestas que aporten mecanismos psicológicos, para realmente conducir al presente y futuro diseñador a crear conciencia, entendimientos emocionales, relaciones, y conexiones, que lo guíen a internalizar la experiencia de otra persona, a ser realmente empático con ella e ir más allá de esa observación y cuestionamiento del usuario como un ente ajeno o distinto al propio diseñador, siendo que es igualmente un individuo, un ser humano, una persona, un usuario.

\section{Comentarios finales}

Con base en la experiencia didáctica desarrollada, a continuación se expone la utilidad e importancia actual y potencial para la formación del estudiante al estimular la empatía.

a En primer lugar, el punto de partida es el alumno, el ser empático con base en la introspección, les conduce a conocerse y entenderse a sí mismos y por lo tanto les brinda la posibilidad de experimentar un crecimiento personal en ese sentido. 
b Abre la posibilidad de una mayor sensibilidad a problemas comunes por parte del alumno, lo cual podría conducir con el tiempo a fomentar una mayor cohesión social, a abrir la posibilidad de que dejen de lado prejuicios y valores relativos, al igual que discriminaciones de todo tipo.

c A nivel disciplinar, y considerando temáticas que en la actualidad se abordan cada vez más frecuentemente y que cobran mayor importancia por su relevancia y congruencia con la naturaleza humana, fomentar la integración de la empatía en la proyección del diseño ayuda a acercarse y comenzar a conocer dimensiones de usuario tan complejas como lo es la emocional y afectiva.

De este modo, la enseñanza de la empatía, a partir de la introspección del alumno, como un primer paso, representa un gran reto en la actualidad, por lo cual resulta enormemente relevante explorar procesos de enseñanza que completen una estrategia didáctica hacia el desarrollo de esta habilidad y de este modo conduzcan y enseñen a las nuevas generaciones a ser empáticos con los usuarios del diseño, actuales o futuros, para contribuir a la construcción de un diseño más humano y quizás con un mayor impacto social. 


\section{Referencias}

Battarbee. K. \& Koskinen, I. (2005). Co-experience: user experience as interaction. CoDesign, vol 1 (1), 5-18. Recuperado de: https://blogs.aalto.fi/ stratusreader/2011/04/05/battarbee-k-and-koskineni-2005-co-experience-user-experience-as-interaction/

Battarbee, K. (2004). Co-experience: understanding user experience in social interaction. Doctoral dissertation. Series ILMARI A51, University of Art and Design Helsinki

Bedolla-Pereda D. (2002). Diseño sensorial las nuevas pautas para la innovación, especialización y personalización del producto. Tesis de Doctorado, Universidad Politécnica de Cataluña (UPC), Barcelona. Recuperado de: http://www.tdx.cat/ handle/10803/6826 ISBN: 84-688-0748-6 D.L: B-4730-2003

Bloom P. (2016). Against empathy, the case for rational compassion, Post best book, New York.

Brown, Tim \& J. Wyatt (2010.) Design Thinking and Social Innovation, Stanford Social Innovation Review.

Buchenau, M. \& Fulton-Suri, J. (2000). Experience prototyping. En: Boyarski, D. \& Kellogg W.A., (Eds.) Proceedings of the conference on designing interactive systems: processes, practices, methods, and techniques, 17-19 August. New York, NY: ACM Press.

Cooper, B. (2004). Empathy, Interaction and Caring: Teachers' Roles in a Constrained Environment. Pastoral Care in Education, 22 (3).

Cote V. \& Gagnon C., (2014). Learning from others: A five years' experience on teaching empathic design. Recuperado de: http://www.drs2014.org/ media/654157/0222-file1.pdf

Desmet, P. M. A. (2002). Designing Emotions. Delft University of Technology, Delft, Holanda

Erikson, E. (1983). Infancia y sociedad. Buenos Aires: Horme-Paidós.
Fulton-Suri, J. (2003). Empathic design: informed and inspired by other people's experience. En: Koskinen, I.; Battarbee, K. \& Mattelmäki T. (Eds.). Empathic design, user experience in product design. Helsinki: IT Press.

García, E. (2008). Neuropsicología y educación, de las neuronas espejo a la teoría de la mente, Revista de psicología y educación, Vol. 1, 3, (69-90).

Håkansson, J. (2003). Exploring the phenomenon of empathy. Doctoral dissertation. Stockholm University, Department of Psychology, Stockholm.

Keller, I. \& Stappers, P.J. (2001). Presence for design: conveying atmosphere through video collages. CyberPsychology and Behavior, 4 (2).

Koskinen, I. \& Battarbee, K. (2003). Introduction to user experience and empathic design. In: Koskinen,I.; Battarbee, K. \& Mattelmäki, T. (Eds.). Empathic design, user experience in product design. Helsinki: IT Press.

Koskinen, I., Battarbee, K., \& Mattelmäki, T. (2003). Empathic design, user experience in product design. Helsinki: IT Press.

Laurel, B. (2003). Design research: methods and perspectives. Cambridge, MA: MIT Press.

van der Lelie, C., (2005). The value of storyboards in the product design process. Proceedings of $3 A D$ third international conference on appliance design. Bristol, UK: The Appliance Design Network, 87-89.

Leonard, D. \& Rayport, J.F. (1997). Spark innovation through empathic design. Harvard Business Review, 75 (6).

López-León, R. \& Gómez-Valdez, G. (2014). Aproximaciones a un modelo de seguimiento y evaluación empática para la educación en diseño, Memorias del segundo coloquio Internacional "Las facetas de la Evaluación" UAM Cuajimalpa Ciudad de México. 
López-León, R. (2017). Traspasando las fronteras de la afectividad: los alcances de la empatía en la educación del Diseño, en Ortiz-Nicolás, J.C. (Compilador), Diseño y Afectividad, CIDI UNAM, México.

Mattelmäki, T. \& Battarbee, K., (2002). Empathy probes. En: Binder, T.; Gregory, J. \& Wagner, I. (Eds.) Proceedings of the participatory design conference 2002. Palo Alto CA: CPSR.

McDonagh, D., (2006). Empathic research approaches to support the designer: a supra-qualitative research for designing model. Design Issues.

Kouprie, Merlijn \& Sleeswijk-Visser, Froukje, (2009). A framework for empathy in design: stepping into and out of the user's life, Journal of Engineering Design, Taylor and Francis Group, 20 (5), Octubre.

Morse J., Bartoff J., Anderson G., O'Brien B., Solberg S. (1992). Beyond empathy: Expressions of caring. Journal of Advanced Nursing, 17 (7), 809-821.

Mukamel, R., Ekstrom, Arne D., Kaplan, J., lacoboni, M., Fried, I. (2010), Responses in humans during execution and observation of actions. Current Biology, 20 (8), 750-756.

Nilsson, P., (2003). Empathy and emotions: on the notion of empathy as emotional sharing. Doctoral dissertation. Department of Philosophy and Linguistics, Umeå University, Umeå, Sweden.

Norman A. D., (2004), Emotional design. Why we love (or hate) everyday things, Basic books, New York.

Ortíz-Nicolás, J.C. (2016). Diseñando el cambio. La innovación social y sus retos, Economía Creativa, otoño-invierno 2016 (6), 9-35.

Redolar, D. (2013). Neurociencia Cognitiva, Editorial Médica Panamericana, Madrid, ISBN: 9788498354089.

Reik, T. (1949). Character analysis. Farrar, Strauss, Giroux, New York.
Rifkin, Jeremy. (2010). La civilización empática.

Barcelona, Paidós

Rizzolatti, G., Fogassi, L., \& Gallese, V. (2001). Neurophisiological mechanisms underlying the understanding and imitation of action. Nature Rewiews Neuroscience. 2, 661-670.

Rogers, C.R., (1975). Empathic: an unappreciated way of being. Counseling Psychologist, 5 (2).

Rogers, C.R., (1961). On Becoming a Person: A Therapist's View of Psychotherapy. New York: Houghton Mifflin Company.

Sanders, E.B.-N. \& Dandavate, U. (1999). Design for experiencing: new tools. En: Overbeeke, C.J. \& Hekkert, P. (Eds.). Proceedings of the first international conference on design and emotion, The Netherlands:Delft University of Technology, Delft.

Stein, E. (1917). Zum Problem der Einfühlung. Halle:Waisenhauses.

Stojiljkovic, S., Dijigic, G., Slatkovic, B. (2012). Empathy as Teacher Roles. Procedia-Social and Behavioral Sciences, 69 (2012), 960-966.

Villegas-Besora, Manuel. (1986). Anuario de psicología: La psicología humanista, historia, concepto y método, núm. 34, Facultad de Psicología Universidad de Barcelona.

Tomberg, Vladimir. \& Laanpere, Mart. (2014). Teaching design for all through empathic modeling a case study in Tallin University, Estonia, Springer International Publishing, Switzerland.

Wiseman, T. (2007). Toward a Holistic Conceptualization of Empathy for Nursing Practice. Advances in Nursing Science, 30 (3), E61-E72. 